\title{
Extinction by the long dielectric needles
}

\author{
S. L. Cherkas ${ }^{\mathrm{a}}$, N. L. Cherkas ${ }^{\mathrm{b}}$ \\ ${ }^{a}$ Institute for Nuclear Problems, Belarus State University, Bobruiskaya 11, Minsk, 220050 \\ Belarus \\ ${ }^{b}$ Military Academy of the Republic of Belarus, Nezavisimosti Av. 220,Minsk, 220057 \\ Belarus
}

\begin{abstract}
Electromagnetic wave extinction by the very long but finite dielectric needle is compared with that by the infinite dielectric cylinder for an oblique incidence of the electromagnetic wave. It is shown that the renormalized Hankel functions without the logarithmic terms should be used for the calculation of the extinction per unit length of the infinite dielectric cylinder to apply it for extinction calculations by the finite dielectric cylinder.
\end{abstract}

Keywords: scattering by finite dielectric cylinder, scattering amplitude renormalization, extinction by dielectric cylinder, forward scattering amplitude

\section{Introduction}

The problem of the scattering of the electromagnetic wave by the finite dielectric cylinder often arises in the optics and radiophysics. Last time the problem acquires a new aspect in a light of the optical properties of the synthetic nanomediums which contain the long dielectric cylinders or pores as the structure elements [1]. In particular, the matrix of anodized aluminum oxide 2] allows to obtain the arrays of nanorods by filling pores of the matrix by the metals or other substances [3]. The typical problem is a falling of the electromagnetic wave on a layer (or a number of layers) consisting of the parallel dielectric cylinders (nanowires or nanorods) or cylindrical pores. Usually the cylinders are perpendicular to the surface of the layer, and in the general case the electromagnetic wave has an oblique incidence relative to the axis of the cylinders. The typical width of a layer (i.e. length of the cylinder) is of the hundreds of micrometers and the cylinder radius is of order tens nanometers. Thus, the aspect ration is $L /(2 R) \sim 10^{4}$, where $L$ and $R$ are the length and radius of the cylinder respectively. From the other hand, a detector is usually situated at a macroscopical distance from the layer,that is, an every cylinder should be considered as three dimensional object in the scattering problem despite of the large aspect ratio. There is no an analytic solution of the scattering problem

Email address: cherkas@inp.minsk.by (S. L. Cherkas) 
by the finite dielectric cylinder. Moreover, the numerical methods [4, 5, 6] are complicated when the aspect ratio is large. From the other hand, the scattering amplitude by the infinite cylinder is expressed analytically in a closed form [7, 8].

(a)

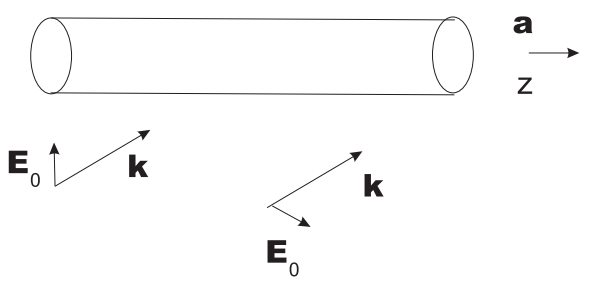

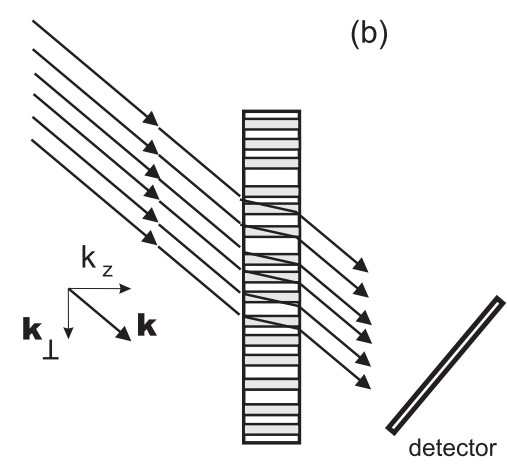

Figure 1: Geometry of the electromagnetic wave scattering by a single dielectric cylinder (a), wave transmission through a layer consisting of the cylinders or cylindrical pores (b).

In the monograph [8] one could read that the formulas for extinction by the infinite cylinder could be applied for the finite one when the boundary effects are negligible. But almost in all the physical situations the boundary effects are considerable! The boundary effects are negligible when an observer is situated at the distance much less then the length of the cylinder, where the field has an asymptotic $1 / \sqrt{r}$, however, generally a finite cylinder has far field falling as $1 / r$ in all direction of the space. Thus, we principally could not avoid the boundary effects if we are talking about the scattering on the three dimensional body.

In Ref. 7, 9] on basis of the Huygens's principle applied at the intermediate zone, where the cylindrical wave transforms into the spherical one, it was argued that under the normal incidence of a wave the extinction cross section by the finite cylinder per unit length equals to that by infinite cylinder. However, the Huygens's principle itself is an approximation.

From the other hand, there exist the approximate methods namely generalized Rayleigh-Gans (GRG) approximation [10, 11] which extends Rayleigh-Gans approximation to the case of the scatterers having one large dimension compared to the others. In the GRG approximation the finite cylinder is considered as a three dimensional object. It seems instructive to compare the extinction in the GRG approximation with that given by the infinite cylinder. That is done in the present paper. Then we suggest a new method how to apply the extinction by the infinite cylinder to finite one.

Certainly there exists the range of the angles where the finite cylinder could not modelled by the infinite one. If one looks along a direction close to the axis of the cylinder it looks like a circle, while the infinite cylinder formally always looks as an extended object. 


\section{GRG approximation}

Let us remind GRG approximation [10, 11] because it will be test bed for comparing with the results for the infinite cylinders.

An electromagnetic field scattered by the dielectric body is expressed with the help of diadic Greene function as 12 ]

$$
\boldsymbol{E}(\boldsymbol{r})=\frac{1}{4 \pi}(\varepsilon-1)\left(\boldsymbol{\nabla} \otimes \boldsymbol{\nabla}+k^{2}\right) \int \frac{\exp \left(i k\left|\boldsymbol{r}-\boldsymbol{r}^{\prime}\right|\right)}{\left|\boldsymbol{r}-\boldsymbol{r}^{\prime}\right|} \boldsymbol{E}\left(r^{\prime}\right) d^{3} \boldsymbol{r}^{\prime}+\boldsymbol{E}_{0} e^{i \boldsymbol{k} \boldsymbol{r}} .
$$

At large $r$ one has

$$
\begin{aligned}
\left(\boldsymbol{\nabla} \otimes \nabla+k^{2}\right) \frac{e^{i k\left|\boldsymbol{r}-\boldsymbol{r}^{\prime}\right|}}{\left|\boldsymbol{r}-\boldsymbol{r}^{\prime}\right|} \boldsymbol{E}\left(\boldsymbol{r}^{\prime}\right) & \approx-k^{2} \frac{e^{i k\left|\boldsymbol{r}-\boldsymbol{r}^{\prime}\right|}}{\left|\boldsymbol{r}-\boldsymbol{r}^{\prime}\right|} \boldsymbol{u} \times\left(\boldsymbol{u} \times \boldsymbol{E}\left(\boldsymbol{r}^{\prime}\right)\right) \\
& \approx-\frac{e^{i k r-i \boldsymbol{k}^{\prime} \boldsymbol{r}^{\prime}}}{r} \boldsymbol{k}^{\prime} \times\left(\boldsymbol{k}^{\prime} \times \boldsymbol{E}\left(\boldsymbol{r}^{\prime}\right)\right),
\end{aligned}
$$

where $\boldsymbol{u}=\frac{\boldsymbol{r}-\boldsymbol{r}^{\prime}}{\left|\boldsymbol{r}-\boldsymbol{r}^{\prime}\right|}, \boldsymbol{k}^{\prime}=k \frac{\boldsymbol{r}}{r}$. In the first approximate equality of the Eq. (2) the terms falling faster then the $\frac{1}{\left|\boldsymbol{r}-\boldsymbol{r}^{\prime}\right|}$ are omitted and in the second approximate equality we set $\boldsymbol{r}-\boldsymbol{r}^{\prime} \rightarrow \boldsymbol{r}$ everywhere except for $\exp \left(i k\left|\boldsymbol{r}-\boldsymbol{r}^{\prime}\right|\right)$ which is approximated as $\exp \left(i k\left|\boldsymbol{r}-\boldsymbol{r}^{\prime}\right|\right) \approx \exp \left(i k r-i \boldsymbol{k}^{\prime} \boldsymbol{r}^{\prime}\right)$.

The next step is to approximate the field $E\left(\boldsymbol{r}^{\prime}\right)$ inside the scatterer. Let us denote the unit vector $\boldsymbol{a}$ along the axis of the cylinder as it it shown in Fig.1 (a). The approximation [10, 11] approachs the field inside the cylinder as uniform in the cross section of the cylinder and depending only on the longitudinal coordinate $z$. This uniform field is applied equal to the static field as though this cylinder would be placed in the external electric field $\boldsymbol{E}_{0}$. That is $\boldsymbol{E}\left(\boldsymbol{r}^{\prime}\right)=$ $\left(\frac{2}{\varepsilon+1}\left(\boldsymbol{E}_{0}-\boldsymbol{a}\left(\boldsymbol{E}_{0} \boldsymbol{a}\right)\right)+\boldsymbol{a}\left(\boldsymbol{E}_{0} \boldsymbol{a}\right)\right) e^{i(\boldsymbol{k a})\left(\boldsymbol{r}^{\prime} \boldsymbol{a}\right)}$. The integral in the Eq. (11) could be calculated and the asymptotic of the scattered field takes the form

$$
\begin{gathered}
\boldsymbol{E}(\boldsymbol{r}) \approx-\frac{e^{i k r}}{r}\left(\boldsymbol{k}^{\prime} \times\left(\boldsymbol{k}^{\prime} \times\left(\frac{2}{\varepsilon+1}\left(\boldsymbol{E}_{0}-\boldsymbol{a}\left(\boldsymbol{E}_{0} \boldsymbol{a}\right)\right)+\boldsymbol{a}\left(\boldsymbol{E}_{0} \boldsymbol{a}\right)\right)\right)\right) \\
\frac{(\varepsilon-1) L R^{2}}{2} \frac{\sin \left(\left(\boldsymbol{k}-\boldsymbol{k}^{\prime}\right) \boldsymbol{a} / 2\right)}{\left(\boldsymbol{k}-\boldsymbol{k}^{\prime}\right) \boldsymbol{a}} .
\end{gathered}
$$

The formula (3) is identical to that of Ref. [10] 1 but it is written purely in the vector form. The differential cross section is defined as

$$
\frac{d \sigma}{d \Omega}=r^{2} \frac{\boldsymbol{E}(\boldsymbol{r}) \boldsymbol{E}^{*}(\boldsymbol{r})}{E_{0}^{2}},
$$

where $d \Omega$ is a cone around $\boldsymbol{k}^{\prime}$.

It is possible to obtain the total cross section in the analytical form [10, 11]. Let us write the cross section for two different cases: an ordinary wave, when

\footnotetext{
${ }^{1}$ Ref. [1] contains misprint in the analogous formula.
} 
the $\boldsymbol{E}_{0}$ is perpendicular to the axis of the cylinder, and an extraordinary wave when it lies in the plane formed by the vectors $\boldsymbol{k}$ and $\boldsymbol{a}$ as it is shown in Fig. 1 (a). In the first case the differential cross section is written as

$$
\begin{aligned}
\sigma_{\perp}=\frac{\pi k^{2} R^{4}(\varepsilon-1)^{2}}{2(\varepsilon+1)^{2}}(2+2 \zeta(\operatorname{Ci}(k L(\zeta+1)-\operatorname{Ci}(k L(1-\zeta))) \\
\left.+\log \left(\frac{2}{\zeta+1}-1\right)\right)-\frac{2 \sin (k L) \cos (k L \zeta)}{k L} \\
+\left(\frac{1+\zeta^{2}}{1-\zeta^{2}}\right)(z \sin (k L) \sin (k L \zeta)+\cos (k L) \cos (k L \zeta)-1) \\
\left.+\left(1+\zeta^{2}\right) k L(\operatorname{Si}(k L(\zeta+1))+\operatorname{Si}(k L-k L \zeta))\right),
\end{aligned}
$$

where $\zeta=(\boldsymbol{k} \boldsymbol{a}) / k=\cos \phi, \mathrm{Ci}$ is the cosine integral function and $\mathrm{Si}$ is sine integral function.

For an extraordinary wave one has

$$
\begin{array}{r}
\sigma_{\|}=\frac{\pi k^{2} R^{4}(\varepsilon-1)^{2}}{2(\varepsilon+1)^{2}}\left((\epsilon+1)^{2}-\zeta^{2}(\epsilon(\epsilon+2)+3)\right)(\zeta(\operatorname{Ci}(k L(1-\zeta)) \\
\left.\left.\left.-\operatorname{Ci}(k L(\zeta+1))+\log \left(\frac{\zeta+1}{1-\zeta}\right)\right)-1\right)+\frac{\sin (k L) \cos (k L \zeta)}{k L}\right) \\
+\left(\zeta^{4}(\epsilon(\epsilon+2)+3)-2 \zeta^{2} \epsilon(\epsilon+2)+(\epsilon+1)^{2}\right) \\
\left(\frac{\zeta \sin (k L) \sin (k L \zeta)+\cos (k L) \cos (k L \zeta)-1}{1-\zeta^{2}}\right. \\
\left.\left.+\frac{k L}{2}(\operatorname{Si}(k L(\zeta+1))+\operatorname{Si}(k L(1-\zeta)))\right)\right) .
\end{array}
$$

One could introduce the extinction cross sections per unit length of the finite cylinder as $C_{\perp G R G}=\sigma_{\perp} / L$ and $C_{\| G R G}=\sigma_{\|} / L$ in order to compare them with that for the infinite one.

\section{Scattering by an infinite cylinder and the amplitude renormaliza- tion}

For an infinite cylinder the extinction cross section is expressed through the forward scattering matrix [7, 8]. Let us write a more general the "off shell" expression for the forward scattering matrix [13]. It arises when a wave propagates in the medium consisting of parallel dielectric cylinders. The positions of the cylinders are suggested to be uncorrelated. The dispersion equation [13] for the effective wave vector $\boldsymbol{k}^{\prime}$ could be put into the form

$$
k^{\prime 2}-k^{2}=4 i n_{0} T\left(\boldsymbol{k}^{\prime}\right)
$$


where $n_{0}$ is the concentration of the centers of the cylinders in a plane perpendicular to the cylinder axis, $k$ is a wave number of the an ectromagnetic wave in vacuum, $T\left(\boldsymbol{k}^{\prime}\right)$ is the "of shell" forward scattering matrix. It is considered that in the random medium there exists the mean field, arising as a result of the multiple scattering of waves [14] by the cylinders. In the Eq. (7) it is taken into account that the wave falling on a cylinder in a random medium differs from that in vacuum, thus, the "off shell" forward scattering amplitude appears [14].
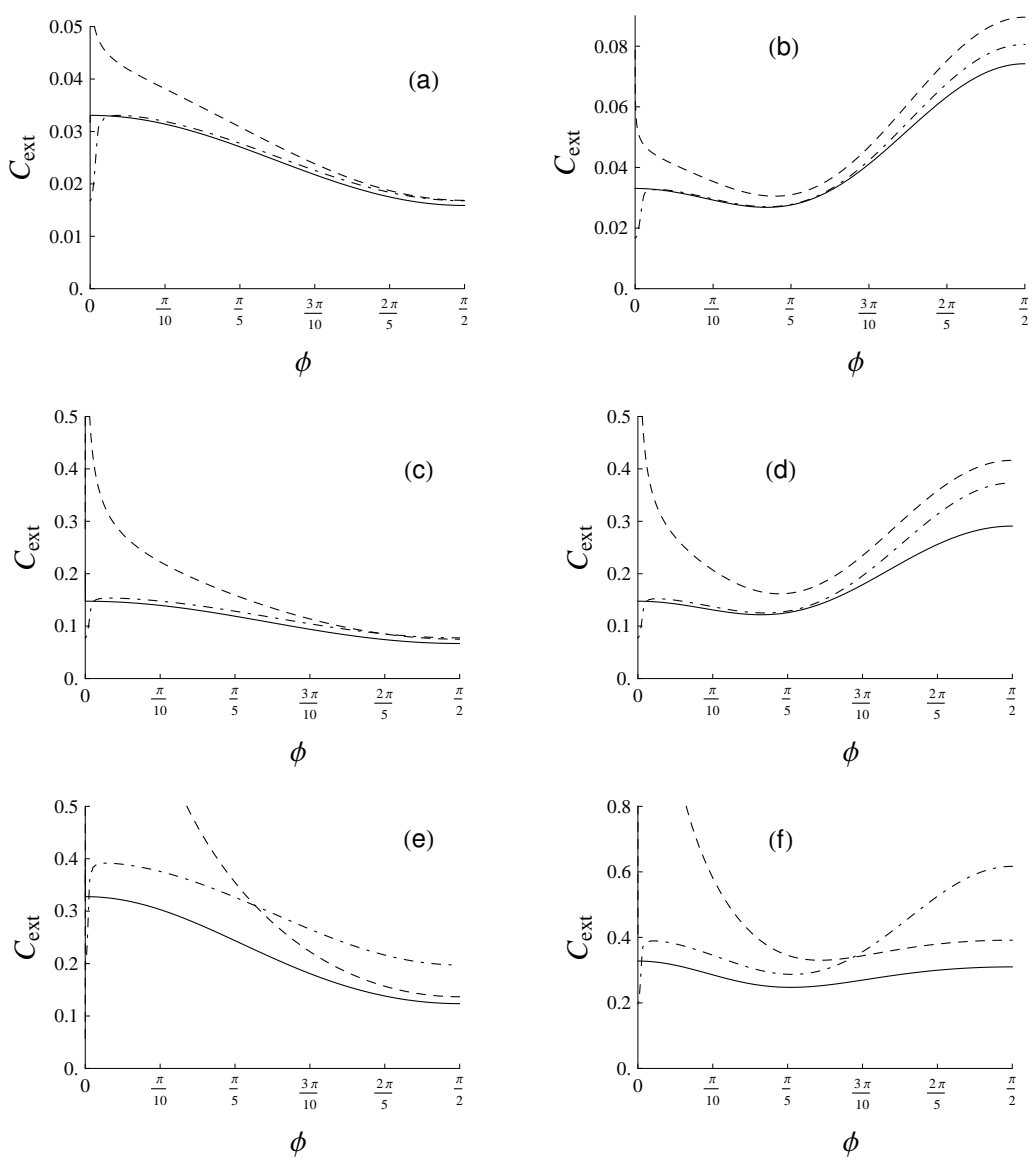

Figure 2: Extinction cross section per unit length of the cylinder $(\mathrm{a}, \mathrm{b})-\varepsilon=2.1, k R=0.3$, $(\mathrm{c}, \mathrm{d})-\varepsilon=2.1, k R=0.5,(\mathrm{e}, \mathrm{f})-\varepsilon=1.5, k R=1$. Figs. (a,c,e) and (b,d,f) correspond to the ordinary and extraordinary waves respectively. Dashed-dotted line - GRG approximation, dashed line - infinite cylinder without renormalization, solid line - renormalized extinction by the infinite cylinder. Aspect ratio $L /(2 R)=10^{4}$ for GRG, $\phi$ is the angle between wave number $\boldsymbol{k}$ and axis of the of the cylinder.

In particular, if the concentration $n_{0}$ is low

$$
\boldsymbol{k}^{\prime} \approx \boldsymbol{k}\left(1+2 i n_{0} T(\boldsymbol{k}) / k^{2}\right)
$$

where $T(\boldsymbol{k})$ is the "on shell" value of the forward scattering matrix $T\left(\boldsymbol{k}^{\prime}\right)$ when 
$\boldsymbol{k}^{\prime}$ is applied equal to $\boldsymbol{k}$. From Eq. (8) it follows $\operatorname{Im} k^{\prime}=C_{\text {inf }} n_{0} / 2$, where

$$
C_{\text {inf }}=\frac{4}{k} \operatorname{Re}[T(\boldsymbol{k})]
$$

is the extinction cross section per unit length of the infinite cylinder. According to (8), (9) the intensity of the wave decreases as the $I=I_{0} \exp \left(-C_{\text {inf }} n_{0} l\right)$ when the wave propagates in a random medium consisting of parallel dielectric cylinders. Here $l$ is the path length in the $\boldsymbol{k}$ direction.

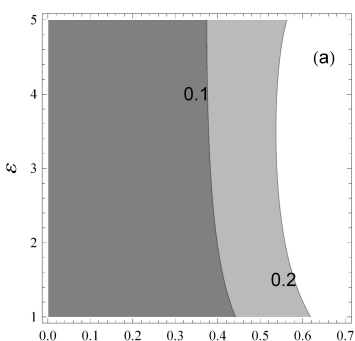

$\mathrm{kR}$

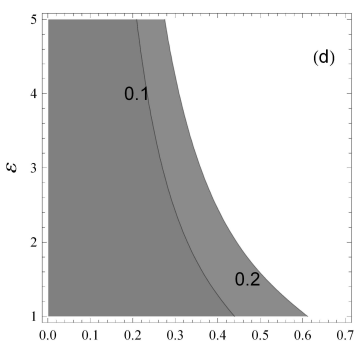

$\mathrm{kR}$

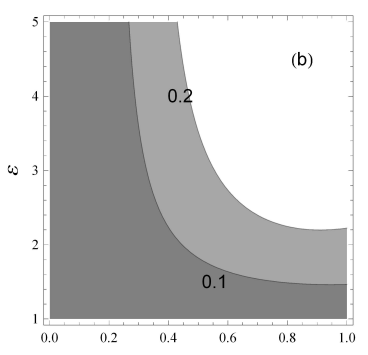

$\mathrm{kR}$

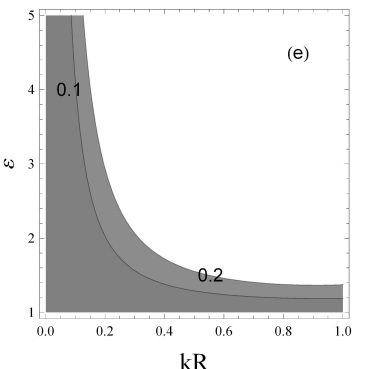

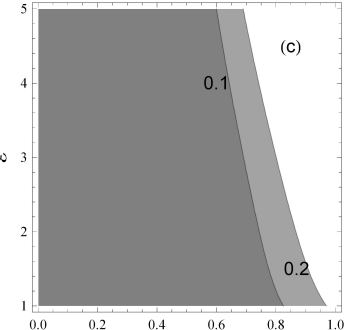

$\mathrm{kR}$

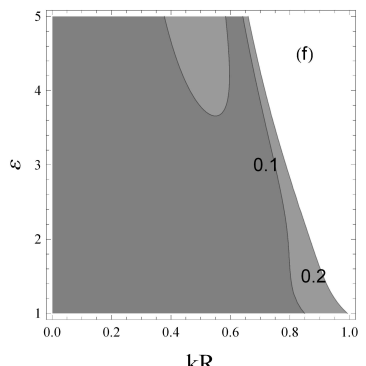

Figure 3: The ranges where the extinction errors of the various approximations do not exceed 10 and 20 percents relative to the renormalized extinction by infinite cylinder (a), (d) -for GRG approximation, (b),(e) - for an infinite cylinder without renormalization, (c),(f) - the expansion to the series given by Eqns. 15, (16). Figs. (a),(b),(c) correspond to an ordinary wave, (d),(e),(f) correspond to an extraordinary wave. The aspect ratio is $L /(2 R)=10^{4}$ for GRG. The results is for the normal wave falling to the cylinder.

In the general case "off shell" expressions for the forward scattering matrix is given by [13]

$$
T_{\perp}\left(\boldsymbol{k}^{\prime}\right)=\frac{\pi}{4 i} \sum_{n=0}^{\infty} \frac{\chi_{n}^{-} \frac{k_{z}^{\prime}}{k}\left(\delta_{n}^{+} \alpha_{n}+\frac{k_{z}^{\prime}}{k} \delta_{n}^{-} \beta_{n}\right)+\chi_{n}^{+}\left(\delta_{n}^{+} \gamma_{n}+\frac{k_{z}^{\prime}}{k} \delta_{n}^{-} \alpha_{n}\right)}{\mathcal{D}_{n}}
$$

for an ordinary wave and

$T_{\|}\left(\boldsymbol{k}^{\prime}\right)=\frac{\pi}{4 i} \sum_{n=0}^{\infty} \frac{\chi_{n}^{+} \frac{k_{z}^{\prime}}{k}\left(\delta_{n}^{-} \alpha_{n}+\frac{k_{z}^{\prime}}{k} \delta_{n}^{+} \beta_{n}\right)+\chi_{n}^{-}\left(\delta_{n}^{-} \gamma_{n}+\frac{k_{z}^{\prime}}{k} \delta_{n}^{+} \alpha_{n}\right)+\chi_{n}^{0} \delta_{n}^{0} \beta_{n}}{\mathcal{D}_{n}}$ 
for an extraordinary wave. It is implied that $z$-axis is directed along the axis $\boldsymbol{a}$ of a cylinder, i.e. $k_{z}^{\prime}$ is the component of $\boldsymbol{k}^{\prime}$ parallel to $\boldsymbol{a}$ and $\boldsymbol{k}_{\perp}^{\prime}$ is perpendicular to $\boldsymbol{a}$ as it shown in Fig. 1 (b).

Other notations in the Eqs. (10), (11) are

$$
\begin{aligned}
& \alpha_{n}=n \frac{k_{z}^{\prime}}{k}\left(\frac{\lambda^{2}}{v^{2}}-1\right) H_{n}(v R) J_{n}(\lambda R), v=\sqrt{k^{2}-k_{z}^{\prime 2}}, \lambda=\sqrt{\varepsilon k^{2}-k_{z}^{\prime 2}}, \\
& \beta_{n}=\lambda R\left(\frac{\lambda}{2 v}\left(H_{n-1}(v R)-H_{n+1}(v R)\right) J_{n}(\lambda R)-H_{n}(v R) J_{n}^{\prime}(\lambda R)\right), \\
& \gamma_{n}=\lambda R\left(\frac{\lambda}{2 v}\left(H_{n-1}(v R)-H_{n+1}(v R)\right) J_{n}(\lambda R)-\varepsilon H_{n}(v R) J_{n}^{\prime}(\lambda R)\right), \\
& \delta_{n}^{ \pm}=\frac{\lambda k^{2}(\varepsilon-1)}{v\left(\lambda^{2}-k_{\perp}^{\prime 2}\right)}\left(k_{\perp}^{\prime} R H_{n+1}(v R) J_{n+1}^{\prime}\left(k_{\perp}^{\prime} R\right)\right. \\
& -\frac{v R}{2}\left(H_{n}(v R)-H_{n+2}(v R)\right) J_{n+1}\left(k_{\perp}^{\prime} R\right) \pm\left(k_{\perp}^{\prime} R H_{n-1}(v R) J_{n-1}^{\prime}\left(k_{\perp}^{\prime} R\right)\right. \\
& \left.\left.-\frac{v R}{2}\left(H_{n-2}(v R)-H_{n}(v R)\right) J_{n-1}\left(k_{\perp}^{\prime} R\right)\right)\right), \\
& \delta_{n}^{0}=\frac{2(\varepsilon-1) \lambda^{2}}{\lambda^{2}-k_{\perp}^{\prime 2}}\left(k_{\perp}^{\prime} R H_{n}(v R) J_{n}^{\prime}\left(k_{\perp}^{\prime} R\right)\right. \\
& \left.-\frac{v R}{2}\left(H_{n-1}(v R)-H_{n+1}(v R)\right) J_{n}\left(k_{\perp}^{\prime} R\right)\right), \\
& \chi_{n}^{ \pm}=\left\{\begin{array}{l}
\left(k_{\perp}^{\prime} R J_{n+1}(\lambda R) J_{n+1}^{\prime}\left(k_{\perp}^{\prime} R\right)-\lambda R J_{n+1}\left(k_{\perp}^{\prime} R\right) J_{n+1}^{\prime}(\lambda R)\right) \\
\pm\left(k_{\perp}^{\prime} R J_{n-1}(\lambda R) J_{n-1}^{\prime}\left(k_{\perp}^{\prime} R\right)-\lambda R J_{n-1}\left(k_{\perp}^{\prime} R\right) J_{n-1}^{\prime}(\lambda R)\right), n \neq 0, \\
(1 \pm 1) / 2\left(k_{\perp}^{\prime} R J_{1}(\lambda R) J_{1}^{\prime}\left(k_{\perp}^{\prime} R\right)-\lambda R J_{1}\left(k_{\perp}^{\prime} R\right) J_{1}^{\prime}(\lambda R)\right), n=0,
\end{array}\right. \\
& \chi_{n}^{0}=\left\{\begin{array}{l}
2\left(k_{\perp}^{\prime} R J_{n}(\lambda R) J_{n}^{\prime}\left(k_{\perp}^{\prime} R\right)-\lambda R J_{n}\left(k_{\perp}^{\prime} R\right) J_{n}^{\prime}(\lambda R)\right), n \neq 0, \\
\left(k_{\perp}^{\prime} R J_{0}(\lambda R) J_{0}^{\prime}\left(k_{\perp}^{\prime} R\right)-\lambda R J_{0}\left(k_{\perp}^{\prime} R\right) J_{0}^{\prime}(\lambda R)\right), n=0,
\end{array}\right. \\
& \mathcal{D}_{n}=\alpha_{n}^{2}-\beta_{n} \gamma_{n} \text {. }
\end{aligned}
$$

For the "on shell" scattering, when $k^{\prime}=\sqrt{k_{z}^{\prime 2}+\boldsymbol{k}_{\perp}^{\prime 2}}=k$, the amplitudes $T_{\perp}\left(\boldsymbol{k}^{\prime}\right)$ and $T_{\|}\left(\boldsymbol{k}^{\prime}\right)$ are reduced to the amplitudes $T_{\perp}(\boldsymbol{k})$ and $T_{\|}(\boldsymbol{k})$ describing forward light scattering by a single infinite cylinder. They presented, for instance, in Refs. [7, 8]. Let us calculate numerically the extinction cross section divided by the length of the cylinder in the GRG approximation (dashed-doted lines in Fig. 21) and compare it with that for infinite cylinder (dashed lines in 
Fig. (2). As one can see from Fig. 2 the cross sections are close near the perpendicular incidence and differ substantially when the direction of the incident wave is close to the axis of the cylinder. There exists the narrow gap, in the GRG approximation related to the finiteness of the cylinder, however, even outside of this gap the curves remains far one from another. Moreover, the extinction cross section for the infinite cylinder diverges logarithmically in the vicinity of small $k_{\perp}$. As for the GRG approximation, it does not contain logarithmic terms asymptotically at $L \rightarrow \infty$ and $k_{\perp}>>1 / L$. This observation leads to an idea of removing all the logarithmic terms from the forward scattering amplitude by the infinite cylinder. Fortunately it is easy to do because the logarithmic terms originate from the Hankel functions. According to [15] the Hankel function of the first kind could be represented in the form

$$
H_{n}(z)=J_{n}(z)+i Y_{n}(z)
$$

where $J_{n}(z), Y_{n}(z)$ are the Bessel functions of the first and the second kind respectively. Regular part of the function $Y_{n}(z)$ could be extracted with the help of the formula [15]

$$
\begin{gathered}
\pi Y_{n}(z)=2 J_{n}(z)\left(\ln \frac{z}{2}+\gamma\right)-\sum_{k=0}^{n-1} \frac{(n-k-1) !}{k !}\left(\frac{z}{2}\right)^{2 k-n} \\
-\left(\frac{z}{2}\right)^{n} \frac{1}{n !} \sum_{k=1}^{n} \frac{1}{k}-\sum_{k=1}^{\infty} \frac{(-1)^{k}}{k !(k+n) !}\left(\frac{z}{2}\right)^{n+2 k}\left(\sum_{m=1}^{n+k} \frac{1}{m}+\sum_{m=1}^{k} \frac{1}{m}\right),
\end{gathered}
$$

where $\gamma$ is Euler constant. As a result, renormalized Hankel function without logarithmic terms is

$$
H_{n}^{r e n}(z)=J_{n}(z)+i\left(Y_{n}(z)-\frac{2}{\pi} J_{n}(z) \ln \frac{z}{2}\right) .
$$

Let us calculate the "on shell" forward scattering matrix and corresponding extinction cross section using formulas (10), (11) and renormalized Hankel function (14). It should be emphasized that before the renormalization the amplitudes (10), (11) have been written in the form containing Hankel functions but not their derivatives.

As it is shown in Fig 2 the coincidence with the extinction in the GRG approximation and that given by the infinite cylinder after renormalization (14) is rather well in a range where GRG have to be valid. For an extraordinary wave the coincidence is not so well, but probably, it is related with the applicability of the GRG approximation.

Let us put the forward scattering amplitudes (10), (11) "on shell" and expand them into the series up to the terms of the six order in $R$. Representing the 
resulting amplitudes as $T_{\text {ser }}=T_{\text {ser }}^{\prime}+i T_{\text {ser }}^{\prime \prime}$ we come to

$$
\begin{gathered}
T_{\perp \text { ser }}^{\prime}=\frac{\pi^{2}(k R)^{4}(\epsilon-1)^{2}}{32(\epsilon+1)^{3}}\left(( k R ) ^ { 2 } \left(2 \zeta^{2}\left(\epsilon^{2}-1\right)+\zeta^{4}(\epsilon+3)\right.\right. \\
\left.\left.-4 \gamma\left(\zeta^{2}+1\right)^{2}(\epsilon-1)-\epsilon-3\right)+4\left(\zeta^{2}+1\right)(\epsilon+1)\right) \\
T_{\perp \text { ser }}^{\prime \prime}=-\frac{\pi(k R)^{2}(\epsilon-1)}{384(\epsilon+1)^{3}}\left(( k R ) ^ { 4 } ( \epsilon - 1 ) \left(-\zeta^{2}+24 \gamma\left(-2 \zeta^{2}\left(\epsilon^{2}-1\right)\right.\right.\right. \\
\left.-\zeta^{4}(\epsilon+3)+\epsilon+3\right)+\zeta^{4}\left(23 \epsilon-3 \epsilon^{2}+20\right)+\zeta^{2} \epsilon\left(2 \epsilon^{2}-\epsilon-16\right) \\
\left.-12 \pi^{2}\left(\zeta^{2}+1\right)^{2}(\epsilon-1)+48 \gamma^{2}\left(\zeta^{2}+1\right)^{2}(\epsilon-1)+\epsilon\left(2 \epsilon^{2}+8 \epsilon-11\right)-23\right) \\
\left.+12(k R)^{2}\left(\epsilon^{2}-1\right)\left(\zeta^{2}(\epsilon-8 \gamma-1)+\epsilon-8 \gamma+3\right)+192(\epsilon+1)^{2}\right) .
\end{gathered}
$$
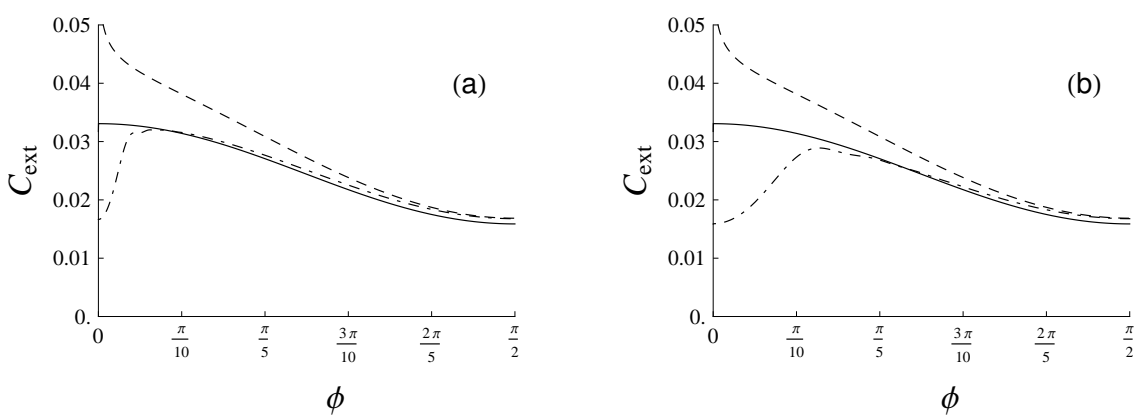

Figure 4: The same as in Fig. 2 (a) but for the aspect ratio used in GRG $L /(2 R)=10^{3}$ (a), $L /(2 R)=10^{2}-(\mathrm{b})$.

For an extraordinary wave one have

$$
\begin{gathered}
T_{\| s e r}^{\prime}=\frac{\pi^{2}(k R)^{4}(\epsilon-1)^{2}}{64(\epsilon+1)^{3}}\left(( k R ) ^ { 2 } \left(4 \gamma \left(\zeta^{6}+7 \zeta^{4}-\zeta^{2}+\left(\zeta^{2}-1\right)^{3} \epsilon^{4}\right.\right.\right. \\
\left.+2\left(\zeta^{2}-1\right)^{3} \epsilon^{3}+2\left(-2 \zeta^{6}+\zeta^{4}-4 \zeta^{2}+1\right) \epsilon+1\right) \\
+\zeta^{6}\left(3 \epsilon^{3}+9 \epsilon^{2}+15 \epsilon+13\right)+\zeta^{4}(\epsilon+1)\left(\epsilon^{3}-7 \epsilon^{2}-17 \epsilon-17\right) \\
\left.+\zeta^{2}\left(3-2 \epsilon^{4}+3 \epsilon^{3}+25 \epsilon^{2}+27 \epsilon\right)+(\epsilon-3)(\epsilon+1)^{3}\right) \\
\left.+4(\epsilon+1)\left(\zeta^{4}\left(\epsilon^{2}+2 \epsilon+3\right)-2 \zeta^{2} \epsilon(\epsilon+2)+(\epsilon+1)^{2}\right)\right),
\end{gathered}
$$




$$
\begin{gathered}
T_{\| s e r}^{\prime \prime}=\frac{\pi(k R)^{2}(\epsilon-1)}{384(\epsilon+1)^{3}}\left(( k R ) ^ { 4 } ( \epsilon - 1 ) \left(\zeta ^ { 4 } \left(6 \pi^{2}\left(\epsilon\left(3 \epsilon^{3}+6 \epsilon^{2}-2\right)-7\right)\right.\right.\right. \\
\left.+\epsilon\left(38 \epsilon^{2}+101 \epsilon+72\right)-3\right)+\zeta^{6}\left(-\left(6 \pi^{2}\left(\epsilon^{4}+2 \epsilon^{3}-4 \epsilon+1\right)\right.\right. \\
\left.\left.+\epsilon\left(12 \epsilon^{2}+31 \epsilon+43\right)+18\right)\right)+\zeta^{2}\left(6 \pi^{2}\left(-3 \epsilon^{4}-6 \epsilon^{3}+8 \epsilon+1\right)\right. \\
\left.+\epsilon\left(2 \epsilon\left(\epsilon^{2}-17 \epsilon-49\right)-53\right)+15\right)+24 \gamma^{2}\left(\zeta^{6}+7 \zeta^{4}-\zeta^{2}+\left(\zeta^{2}-1\right)^{3} \epsilon^{4}\right. \\
\left.+2\left(\zeta^{2}-1\right)^{3} \epsilon^{3}+2\left(-2 \zeta^{6}+\zeta^{4}-4 \zeta^{2}+1\right) \epsilon+1\right)+12 \gamma\left(\zeta ^ { 6 } \left(3 \epsilon^{3}+9 \epsilon^{2}\right.\right. \\
+15 \epsilon+13)+\zeta^{4}(\epsilon+1)\left(\epsilon\left(\epsilon^{2}-7 \epsilon-17\right)-17\right)+\zeta^{2}\left(\epsilon \left(\left(3 \epsilon^{2}-2 \epsilon^{3}+25 \epsilon\right)\right.\right. \\
\left.\left.+27)+3)+(\epsilon-3)(\epsilon+1)^{3}\right)+2\left(3 \pi^{2}(\epsilon-1)-\epsilon+5\right)(\epsilon+1)^{3}\right) \\
+12(k R)^{2}\left(\epsilon^{2}-1\right)\left(\zeta^{4}(3 \epsilon+4 \gamma(\epsilon(\epsilon+2)+3)+5)+\zeta^{2}(\epsilon(\epsilon-8 \gamma(\epsilon+2)-3)\right. \\
\left.\left.-6)+(4 \gamma-1)(\epsilon+1)^{2}\right)+96(\epsilon+1)^{2}\left(\zeta^{2}(\epsilon-1)-\epsilon-1\right)\right) .
\end{gathered}
$$

The equations (15), (16), (17), (18) do not contain any logarithmic terms because they were removed by the renormalization of the Hankel function. In comparison with the extinction, given by the GRG approximatioin, the equations (15), (17) contain the terms of the six order in $R$ and, thus, they have the wider applicability for the shorter wavelength.

In the general case, if to consider falling of the electromagnetic wave on the layer of cylinders not only the extinction cross section is needed but the effective refractive index of a layer. It can be found from the dispersion equation for the wave vector (7) using renormalized Hankel functions.

\section{Discussion and conclusion}

The expressions (15), (16), (17), (18) are of the six order in $R$. The expressions (5), (6) are of the fourth order in $R$. Calculating the asymptotic of the $\sigma / L$ given by (5), (6) in the limit $L \rightarrow \infty, k_{\perp}>>1 / L$ we have found that the extinctions per unit length of the finite cylinder coincides exactly with the corresponding $R^{4}$ terms contained in the Eq. (15), (17).

The fascinating hypothesis arises that in the limit $L \rightarrow \infty, k_{\perp}>>1 / L$ the extinction cross section as a result of the exact solution of the three dimensional scattering problem coincides exactly, i.e. in the all orders in $R$, with the extinction by the infinite cylinder obtained with the renormalized Hankel functions. From physical point of view it means that with the help of renormalization of the Hankel functions one is able to describe correctly the extinction by the long but finite dielectric cylinder in all orders in $k R$ and in all the range of the incidence angles excluding the narrow gap near $\phi=0$.

We have no possibility to check this general hypothesis analytically, thus, we have presented numerical results showing the ranges, where the relative errors 
compared to $G R G$ and the infinite cylinder without renormalization approximations do not exceed 10 per and 20 percents. The normal incidence of a wave to the cylinder is considered in order to check also the earlier hypothesis of van der Hulst [7, 9] that at a normal wave incidence an extinction by the finite cylinder is equal to that by the infinite cylinder (without renormalization). As one could see the hypothesis of van der Hulst is approximate, because the logarithmic terms spoil the coincidence even at the normal incidence, when the dielectric constant and $k R$ become large. At the same time from the Fig. 3 we see that the GRG approximation and the approximate formulas (15), (17) work at a relatively wide range.

As it was mentioned the gap for the small angles of incidence relative the axis of the finite cylinder could not principally be described in a frame of scattering by infinite cylinder. From the Fig. 4 one could see this gap broadening with the rising of the aspect ratio. However, at low aspect ratio the numerical methods can be easily applied.

We may conclude that the renormalized Hankel functions should be used to describe the extinction as by a single cylinder so in the dispersion equations for the effective wave number (77). The dispersion equation (7) could be applied straightforwardly to the mediums where the correlation in the placement of the scatterers are absent, for instance, porous silicon [17]. However, for instance, for porous aluminium oxide [16] the correlation of the scatterers should be taken into account [18] with the help of radial distribution function [19].

\section{References}

[1] G. D. Sulka, Highly Ordered Anodic Porous Alumina Formation by SelfOrganized Anodizing, in: Nanostructured Materials in Electrochemistry / Ed. by A. Eftekhari, P. 1, (Wiley-VCH, Weinheim, 2008).

[2] L. K. Hudson , C. Misra , A. J. Perrotta et al, Aluminum oxide. (WileyVCH, Weinheim, 2000).

[3] J.-M. Moon and A. Wei, Uniform gold nanorod arrays from polyethylenimine-coated alumina templates, J. Phys. Chem. B 109 (2005) 23336 .

[4] V. G. Vereshchagin, R. A. Dynich and A. N. Ponyavina, J. Appl. Spectr., Radiation scattering by finite dielectric cylinders, 65 (1998) 267.

[5] T. Rother, S. Havemann and K. Schmidt, Scattering of plane waves on finite cylinders with non-circular cross-section. Progress In Electromagnetics Research, 23, (1999) 79 .

[6] W.Z. Yan, Y. Du, H. Wu, D.W. Liu and I. Wu, Scattering from a long dielectric circular cylinder, Progress In Electromagnetics Research, 85 (2008) 39. 
[7] H. C. van de Hulst, Light scattering by small particles (John Wiley and Sons, NY, 1957).

[8] C.F. Bohren and D.R. Huffman, Absorption and Scattering of Light by Small Particles (Wiley, NY, 1998).

[9] Ru.T. Wang and H.C. van de Hulst, Appl. Opt. 34 (1995) 2811.

[10] R. Schiffer and K. O. Thielheim, Light scattering at dielectric needles and circular disks, Astronomische Gesellschaft, Mitteilungen, 43 (1978) 289.

[11] R. Schiffer and K. O. Thielheim, Light scattering by dielectric needls and disks, J. Appl. Phys. 50 (1979) 2476.

[12] J.D. Jackson, Classical Electrodynamics, (John Wiley and Sons, NY, 1962).

[13] N.L. Cherkas, dispersion equations for the wave vector of an electromagnetic wave in a medium consisting of parallel dielectric cylinders, Differential Equations, 35 (1999). 1160.

[14] M. Lax, Multiple Scattering of Waves. II. The Effective Field in Dense Systems, Phys. Rev. 85 (1952) 621.

[15] I.S. Gradshtein and I.M. Ryzhik, Table of Integrals, Series, and Products (Academic Press, NY, 1994).

[16] A.A. Lutich et al, Anisotropic Light Scattering in Nanoporous Materials: A Photon Density of States Effect, Nano Letters 4 (2004) 1755.

[17] A.E. Pap, K. Kordas et al, Optical properties of porous silicon. Part III: Comparison of experimental and theoretical results, Optical Materials 28(2006) 506.

[18] N.L. Cherkas, Electromagnetic wave in a medium composed of parallel dielectric cylinders, Opt. Spectr. 81(1996) 906.

[19] N.L. Cherkas and S.L. Cherkas, Model of radial distribution function of pores in a layer of porous aluminum oxide, Cryst. Rep. 62 (2016) 285. 